\title{
SUMA: AN INTERFACE FOR SURFACE-BASED INTRA- AND INTER-SUBJECT ANALYSIS WITH AFNI
}

\author{
Ziad S. Saad ${ }^{*}$, Richard C. Reynolds ${ }^{*}$, Brenna Argall ${ }^{\dagger}$ Shruti Japee ${ }^{\dagger}$, Robert W. Cox ${ }^{*}$ \\ * Scientific and Statistical Computing Core, National Institute of Mental Health, National Institutes of Health, Department \\ of Health and Human Services, Bethesda MD, USA. \\ ${ }^{\dagger}$ Laboratory of Brain and Cognition, National Institute of Mental Health, National Institutes of Health, Department of \\ Health and Human Services, Bethesda, MD, USA.
}

\begin{abstract}
Surface-based brain imaging analysis is increasingly being used for detailed analysis of the topology of brain activation patterns and changes in cerebral gray matter. Here we present SUMA, a new interface for visualizing and performing surfacebased brain imaging analysis that is tightly coupled to AFNI - a volume-based brain imaging analysis suite. The interactive part of SUMA is used for rapid and interactive surface and data visualization, access and manipulations with direct link to the volumetric data rendered in AFNI. The batch-mode part of SUMA allows for surface based operations such as geometry and data smoothing [1,2], surface to volume domain mapping in both directions and node-based statistical and computational tools. We also present methods for mapping low resolution functional data onto the cortical surface while preserving the topological information present in the volumetric data and detail an efficient procedure for performing cross-subject, surfacebased analysis with minimal interpolation of the functional data.
\end{abstract}

\section{INTRODUCTION}

Magnetic Resonance Imaging is widely used for noninvasive imaging of brain anatomy and function (FMRI) and much of the research consists of studying the patterns of brain activation or changes in brain anatomy to understand brain function and disease. With MRI methods, the measurement of brain activity, which occurs in neurons forming the grey matter, is sampled using a volumetric grid. Due to the highly convoluted nature of the cortical surface (see Figure 1-A), this sampling can obscure the topological detail of the patterns of brain activation. Partly to address this issue, the volumetric data are mapped onto 3D models of the cortical surface and the topology of activation is analyzed directly on the surface models [3-10]. In addition to recovering the topology of activation, cortical surface models can greatly simplify data visualization; revealing the pattern of activation throughout the cortex with as little as one view (when using flattened surfaces). However, such data representation modes are considerably removed from the original data, which presents two difficulties: data mapping across domains, and relating the various methods of rendering the data. The former problem might affect the topology of activation, and the latter is important from a practical point of view when analyzing complex data. With these difficulties in mind, we designed SUMA, a program that adds cortical surface-based functional imaging analysis to AFNI[11].

SUMA allows viewing of 3D cortical surface models, and mapping of volumetric data onto them. With SUMA, AFNI can simultaneously and in real-time render functional imaging data in 4 modes: Slice, Graph (time series), Volume and Surface, with direct links between them. We begin by presenting the interface's main capabilities followed by a discussion of how volumetric data are mapped onto the surface and a method for normalizing surface meshes which simplifies cross-subject surface-based analysis and surface-based atlas creation/query operations.

\section{SURFACE DATA: VISUALIZATION AND PROCESSING}

SUMA comprises an interactive module and a set of command-line programs. The interactive module is used to visualize surface models and data with the possibility of rendering a multitude of surfaces simultaneously inside one or multiple linked surface viewers. Figure 1 A-C shows three surface viewers displaying surface models of the boundary between gray and white matter (A), an inflated version of it (B), and a flattened representation of the occipital cortex (C). Surface models can be rotated and translated interactively. With the use of common graphics cards supporting hardware-accelerated OpenGL, a surface model with 270,000 triangles is currently rendered in under 0.06 seconds. The interactive modules are also used for manual (free-hand) tracing of Regions Of Interest (ROI) directly on $3 \mathrm{D}$ cortical surface models. Surface viewers can also communicate with AFNI, which provides three modes for rendering volumetric data as shown in Figure 1 D-F. Selecting a location on a surface model in SUMA causes the cross-hair to jump to the corresponding location in the other surface viewers, the volume (D), time series $(\mathrm{E})$, and slice $(\mathrm{F})$ views, and vice versa. Thus statistical parameters shown on the cortical surfaces can be directly linked to the time series data from which they were calculated. In addition, changes in the volumetric functional overlay data are automatically mapped to the surface models and displayed in the surface viewers. Conversely, drawing an ROI on the surface can be directly transformed into an ROI in the volume and displayed in AFNI.

SUMA and AFNI, being separate programs, communicate either through shared memory (if the two programs are running 
on the same computer) or though network sockets. With the large number of data views displayed simultaneously, it is advantageous to run the two programs on separate computers thereby doubling the available screen space. The communication API is named NIML (Neuroimaging Markup Language), which is built on XML (http://www.xml.com) with the main difference being NIML's ability to directly handle binary data.

In addition to the interactive viewers, command-line programs are used to perform batch-mode operations. The most important ones include volume to surface domain mapping and vice versa, surface geometry and data smoothing, and surface thickness and gray matter volume measurements. In addition, voxel-based time series processing, statistical analysis and general purpose computational tools available in AFNI are also applicable to node-based processing.

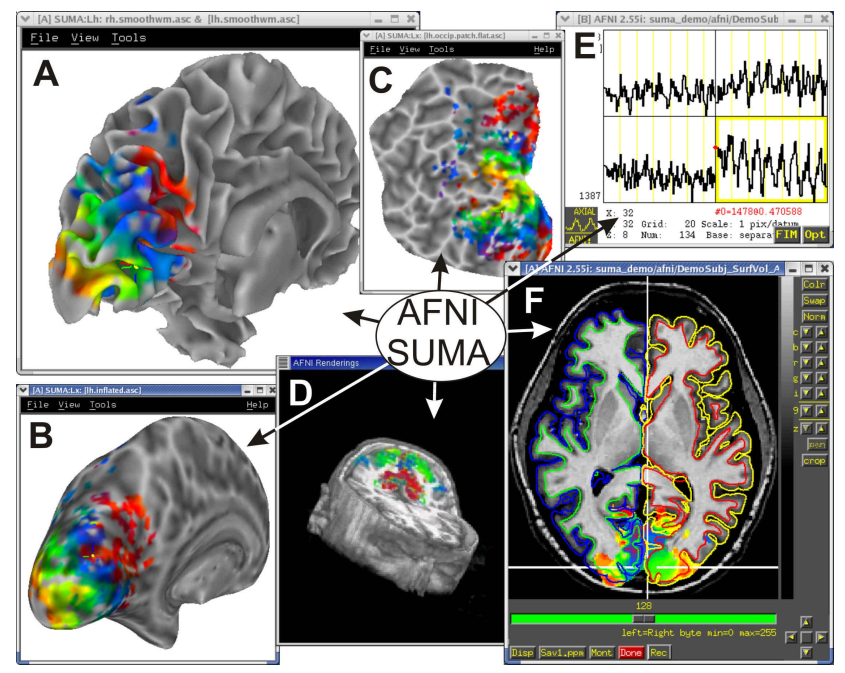

Figure 1: A-Surface viewer displaying a 3D model of the boundary between gray and white matter. B- An inflated version of the model in $A$, revealing the deep sulcal areas. $C$ A flattened version of the occipital lobe in A and B. Gray shading on the three surfaces represents the curvature of the surface in A. D- Volume rendering of volumetric data. ETime series data from voxels in the vicinity of the location of the cross-hair shown in F. F- Slice mode view of volumetric data with the cross-hair positioned in the occipital lobe. All these rendering modes are linked together. On colored reproductions of this figure, colored overlay represents response properties of brain regions showing statistically significant activation.

\section{VOLUME TO SURFACE MAPPING}

Due to the highly convoluted nature of the cortical surface, the topology of activation as it occurred on the cortical sheet is obscured by the volumetric grid used to sample brain activation. In other words, two points A and B shown in Figure 2, may be fairly distant on the cortical surface, but juxtaposed in the volumetric grid. Consequently they may be sampled by the same voxel, or at best, two neighboring voxels that could share highly correlated signal and noise properties. In general, brain locations such as $\mathrm{A}$ and $\mathrm{B}$ are separated in $\mathbf{R}^{3}$ by a distance $R_{A B}$ much smaller than $S_{A B}$, the shortest path between them along the surface $\left(R_{A B} / S_{A B} \ll 1\right)$. To recover the topology of activation, one has to map the activation onto the cortical surface. In the worse case scenario, points A and B may not be distinguishable from each other in the FMRI data, but other, less sensitive, areas would be. It is important to note that such an ambiguity is present in the volumetric data and not a result of the mapping to the cortical surface.

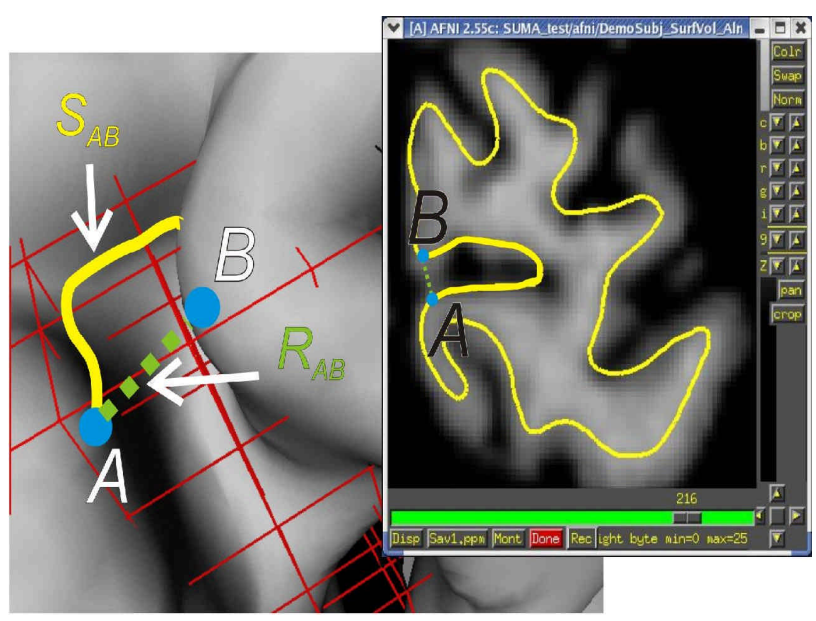

Figure 2: 3D cortical surface model of the occipital cortex approximating cortical layer 4. Points $A$ and $B$ represent two nodes on either side of the calcarine sulcus. $R_{A B}$ and $S_{A B}$ represent the Euclidian distance and the shortest distance along the surface between $A$ and $B$. The red grid represents a typical $4 \mathrm{~mm}$ cubic FMRI sampling grid. The right side shows a coronal slice through the calcarine cortex with the contour of the cortical surface shown in yellow. For clarity, the contour depicts the boundary between the white and grey matter.

Spatial interpolation of the low-resolution volumetric data will further degrade the topological information present in volumetric data. Such interpolations can be avoided by aligning the surface model to the volumetric data instead of the reverse. Should data smoothing be necessary, it can be performed on the data once it is mapped onto the surface [12]. To align the surface model to the volumetric data, we begin by aligning the high-resolution anatomical dataset used to create the surface model to the anatomical dataset obtained during the experiment, then apply the same transform to the surface model. There are no interpolation artifacts or blurring accompanying this alignment since the surface is defined on a continuous coordinate system, unlike the functional data which is defined on a coarse discrete grid. Note also that the alignment performed here does not correct for localized distortions of the functional data relative to the high-resolution anatomy. Such distortions are best corrected during MRI reconstruction. However, if further distortion correction must be made, it should be applied to the surface model rather than to the functional volume prior to mapping the functional data.

Mapping data between surface and volumetric domains is done using the intersection of one surface with the volume or the intersection of a region defined by two isotopic surfaces with the volume. For example, by using surfaces that model inner and 
outer boundaries of the cortical surface, one can map data that intersects the gray matter onto the surface. Conversely, one can turn an ROI defined on the surface into a gray-matter only volume of interest.

\section{STANDARD-MESH SURFACE MODELS}

Current methods for inter-subject surface-based analysis have adopted a spherical coordinate system to allow for spatial normalization of surface maps [6, 13, 14]. Individual brain hemisphere surface models are inflated to a sphere and then warped along the spherical surface to a spherical template, allowing for inter-subject statistical comparisons. However, because discrete surface models from different subjects are often not topologically equivalent, the analysis of inter-subject data requires data interpolation onto a common mesh. This complicates the mapping between surfaces from different subjects and further blurs the topology of activation. The interpolation is necessary because activation maps are attached to the nodes forming the surface (topology) and not to a spatial location (geometry).

Inter-subject surface-based analysis can be greatly simplified by creating a fixed discrete topological frame of reference based in the spherical coordinate system. We replace the meshes of individual surface models with a standard mesh, thereby allowing for node-based correspondence across surface models. The proposed method is independent of surface creation methods and preserves the morphology of the original surfaces. With standard meshes, the index of a node can be used to identify its anatomical location regardless of the surface's geometry or original topology.

Figure 3 illustrates the process of creating a standard-mesh version of an individual subject's anatomically correct surface model (Anat). The first step, illustrated in Figure 3-A, consists of warping Anat to fit a spherical surface template (Template). Anat is first inflated to a sphere ( $\mathrm{Sph}$ ) and then warped (Warp) so that its sulcal patterns match those of the spherical surface template (Template). For reference, the central sulcus is highlighted in all surfaces. Although the warping is done in a distorted space, surface-based warping employs considerably more landmarks than the Talairach transform and the effects of inter-subject anatomical (geometrical) variance are reduced. Surface-based warping offers an advantage over the Talairach normalization because the coordinate warping is confined to and continuous along the surface. In Talairach space, small variations in the $(\mathrm{x}, \mathrm{y}, \mathrm{z})$ coordinates can result in large and discontinuous variations in the activation patterns at points such as $\mathrm{A}$ and $\mathrm{B}$ in Figure 2. Such variations with surface-based warping would be smoother and more localized. The warping in this study was carried out using the spherical warping methods from FreeSurfer package; however, the concept is applicable to any surface-based warping to a template surface.

Next we use the warped surfaces to create standard-mesh surfaces (such as AnatStd) identical in geometry to their original version (such as Anat) but having a standard topology that would be common across surfaces. To create the standard-mesh surfaces, we start with a triangulated icosahedron (IcoStd, Figure 3-B, left) with Nstd nodes and whose co-centered sphere has a radius equal to that of Warp. Each node $n$ of IcoStd, projected radially onto Warp, falls inside a triangle $\mathrm{T}$ of Warp's mesh (Figure 3-B, right). Let $f($.) be a function defined at each node of the Warp surface. The value of $f($.$) at node n$ in the IcoStd surface is computed by interpolation as in Equation 1:

$$
f(n)=a 1 f(n 1)+a 2 f(n 2)+a 3 f(n 3)
$$

where aj is the area (or barycentric) coordinate of node $\mathrm{j}$ of $\mathrm{T}$ and $\mathrm{n} 1, \mathrm{n} 2, \mathrm{n} 3$ are the nodes forming T. The left part of Figure 3-C is a graphical representation of the area coordinates of node $n$ inside T. For example, a1 is the ratio of the area formed by triangle $\mathrm{n}, \mathrm{n} 2, \mathrm{n} 3$ (cross hatching) to the area of $\mathrm{T}$. The use of area coordinates as weights satisfies the continuity condition across the boundaries of T. For example, as node $n$ approaches edge [n2 n3], the contribution of $n 1$ is reduced to 0 . Also, if $n$ approaches $n 2$, the weight for $n 1$ and $n 3$ are reduced to 0 .

Rather than interpolate FMRI data or statistics, we repeatedly replace $f($.$) in Eq. 1$ by the $x(),. y($.$) and z($. coordinates of the Anat surface. In other words, the coordinates of node $\mathrm{n}$ in AnatStd are obtained by interpolating the coordinates of nodes $\mathrm{n} 1, \mathrm{n} 2$, and $\mathrm{n} 3$ in Anat as shown in Figure 3-C. Standard-mesh versions of all other surface models are similarly created. All mapping of functional activity can now be performed on the standard-mesh, anatomically correct surface models instead of the original ones.

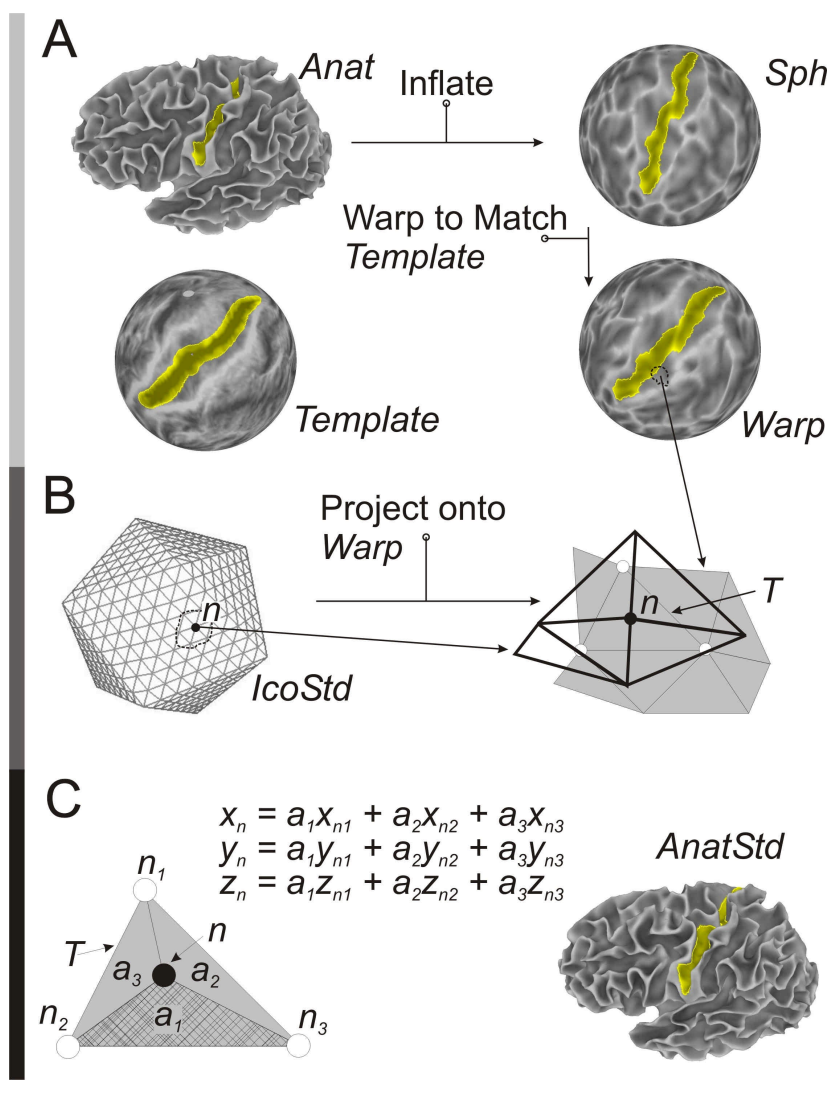

Figure 3: Warping of cortical surface to spherical template. A- Anatomically correct surface model Anat is inflated to a spherical shape (Sph) then warped (Warp) such that its sulcal patterns match those of the spherical template (Template). Template was obtained from the FreeSurfer software package. For reference, the central sulcus is highlighted in all surfaces. B- Illustration of the triangulated icosahedron 
(IcoStd) used to create the standard-mesh surfaces. For clarity, each edge is shown subdivided into 8 parts instead of 141. Each node $n$ of IcoStd is projected onto Warp's spherical mesh (gray shading on the right) thereby intersecting one of Warp's triangles T. For simplicity, this intersection is also referred to by $n$. C- Left: Area (or Barycentric) coordinates a1, a2, and a3 of node $n$ in triangle $\mathrm{T}$ formed by nodes $\mathrm{n} 1, \mathrm{n} 2$, and $\mathrm{n} 3$ from Warp's mesh. a1 is the ratio of the area of the triangle formed by $n, n 2, n 3$ (stripes) to the area of $T$. a 2 and a3 coordinates are similarly calculated with the area of triangle $n, n 2, n 3$ replaced by that of triangles n,n1,n3 and n,n1,n2, respectively. C-Middle: Equations for calculating the coordinates of node $n$ as a function of the coordinates of nodes n1, n2, and $n 3$ in Anat. C-Right: Repeating the previous procedure for all nodes $\mathbf{n}$ forming IcoStd, results in AnatStd, the standard-mesh version of Anat.

On standard meshes, nodes with the same index represent the same anatomical location, within the error of the warping process. Therefore, inter-subject surface-based analysis is reduced to node-based analysis. AFNI's statistical suite of programs designed for voxel-based analysis was easily extended to accommodate node-based statistical analysis.

\section{CONCLUSIONS}

We have presented SUMA, a suite of programs offering a graphical interface for surface models and data visualization and a series of command- line programs for performing surfacebased computations and statistical analysis. The graphical interface is tightly linked to the volumetric data rendered in AFNI thus enabling users to have a direct link between data presentation modes. The process of mapping data across domains was designed to eliminate unnecessary interpolation of volumetric functional data, thereby preserving topological information present in the volumetric data. Lastly, we developed a method for creating a topology-based reference that provides all the functionality of the coordinate-based spherical system while simplifying inter-subject analysis without undue interpolation of the functional data.

AFNI and SUMA are Open Source. Source code, documentation and binaries are freely available from: http://afni.nimh.nih.gov

\section{ACKNOWLEDGMENTS}

Thanks go to R. Doucette for preparation of the manuscript. The authors also wish to thank Drs. Sean Marrett and Hauke Heekeren for optimizing the MPRAGE MRI acquisition and help, along with Dr. P. Christidis, in creating surface models.

\section{REFERENCES}

1. Taubin, G., Curve and surface smoothing without shrinkage. in Fifth International Conference on Computer Vision. 1995.
2. Chung, M.K., et al., Deformation-based surface morphometry applied to gray matter deformation. Neuroimage, 2003. 18(2): p. 198-213.

3. Dale, A.M., et al., Cortical surface-based analysis. I: Segmentation and surface reconstruction. Neuroimage, 1999. 9(2): p. 179-94.

4. Drury, H.A., et al., Computerized mappings of the cerebral cortex: a multiresolution flattening method and a surfacebased coordinate system. Journal of Cognitive Neuroscience, 1996. 8(1): p. 1-28.

5. Fischl, B., et al., Cortical surface-based analysis. II: Inflation, flattening, and a surface-based coordinate system. Neuroimage, 1999. 9(2): p. 195-207.

6. Fischl, B., et al., High-resolution intersubject averaging and a coordinate system for the cortical surface. Hum Brain Mapp, 1999. 8(4): p. 272-84.

7. Kriegeskorte, N. and R. Goebel, An efficient algorithm for topologically correct segmentation of the cortical sheet in anatomical $m r$ volumes. Neuroimage, 2001. 14(2): p. 329-46.

8. MacDonald, D., et al., Automated 3-D extraction of inner and outer surfaces of cerebral cortex from MRI. Neuroimage, 2000. 12(3): p. 340-56.

9. Van Essen, D.C. and H.A. Drury, Structural and functional analyses of human cerebral cortex using a surface-based atlas. Journal of Neuroscience, 1997. 17(18): p. 7079-102.

10. Wandell, B.A., et al., Visualization and measurement of the cortical surface. Journal of Cognitive Neuroscience, 2000. 12(5): p. 739-52.

11. Cox, R.W., AFNI: software for analysis and visualization of functional magnetic resonance neuroimages. Computers \& Biomedical Research, 1996. 29(3): p. 162-73.

12. Andrade, A., et al., Detection of fMRI activation using Cortical Surface Mapping. Hum Brain Mapp, 2001. 12(2): p. 79-93.

13. Van Essen, D.C., et al., Functional and structural mapping of human cerebral cortex: solutions are in the surfaces. PNAS, 1998. 95(3): p. 788-95.

14. Van Essen, D.C., et al., Mapping visual cortex in monkeys and humans using surface-based atlases. Vision Research, 2001. 41(10-11): p. 1359-78. 